\title{
Hybrid order characteristics in car-following behavior
}

\author{
Chunling $\mathrm{Tu}^{1}$, Shengzhi $\mathrm{Du}^{2}$ \\ ${ }^{1}$ Department of Computer Systems Engineering, Tshwane University of Technology, South Africa \\ ${ }^{2}$ Department of Electrical Engineering, Tshwane University of Technology, South Africa
}

\begin{tabular}{l}
\hline \hline Article Info \\
\hline Article history: \\
Received Jan 10, 2020 \\
Revised Mar 12, 2020 \\
Accepted Mar 26, 2020 \\
\hline
\end{tabular}

Keywords:

Car following

Intelligent transport systems

Vehicle behavior modelling

\begin{abstract}
This paper addresses the hybrid order behavior in car-following processes, which was not reported in the existing literatures. The behavior is supported by both experimental data and theoretical simulations. To demonstrate this behavior, the first order and the second order car-following behaviors are defined. By comparing car-following behaviors in the existing analystic models and the real traffic context, this paper finds that a significant amount of the second order car-following processes in real traffic context do not match the models. The structural mismatches suggest the existence of unmodelled dynamics in the existing methods. In fact, the car following behavior is determined by more factors than the immediate proceeding vehicle. Therefore, the existing car-following models must be improved to accommodate these factors. This forms one of the main values of this paper. This paper then defines the hybrid order car-following behavior and prompts to associate this behavior with the concerned unmodelled dynamics. A neural network is employed to model such dynamics. The proposed hybrid order behavior matches the fact that the car-following behavior is determined by multiple vehicles instead of the immediate proceeding one only. This is valuable in providing guidance on the improvement of existing models.
\end{abstract}

Copyright $\odot 2020$ Institute of Advanced Engineering and Science. All rights reserved.

\section{Corresponding Author:}

Shengzhi Du,

Department of Electrical Engineering,

Tshwane University of Technology, Pretoria, South Africa.

Email: dushengzhi@gmail.com

\section{INTRODUCTION}

The road accidents become serious challenges with the rapid increasing of vehicles, which resulted in a large number damages. The global status reported in 2018, there were about 3700 people lost life from the road accident every day, and around tens millions of injury every year. The main contribution for these problems comes from human faults, such as high speed, dangerous overtaking, blind spot mistake, and alcohol or drugs intake. To reduce the road accidents, a variety of technologies and algorithms were developed to understand the traffic flow behavior [1] and assist drivers, for instance, intelligent driver system [2], collision avoidance system [3], and blind spot detection system [4], car-following model [5, 6], and so on.

The car-following model is one of the particular research area for the purpose to control the desired speed, predict the movement of vehicle, and monitor the driving behavior. The car-following model can also be used in self driving vehicles [7] to assit in understanding the behavior of other drivers [8] and the pedestrians [9]. There are numbers of car-following models were performed, which describe how the subject vehicle changes the relative speed and position with the proceeding vehicle. The earliest nonlinear carfollowing models $[5,10]$ were proposed over sixty years. After then, a variety of car-following models [11] were developed that are helpful to determine the acceleration with the consideration of the relative situation (lane, speed and distance with the leading vehicle). A lane changing model was proposed [12] to help drivers make correct lane decision based on predicted driving behaviro of other vehicles, such as the distance, acceleration or deceleration, orientation, and the lane they are using. 
The car-following model can be categorized into physical-based models and learning-based models [13]. The physical-based models are used to present the behavior of drivers with unknown parameter values These parameters have special physical meaning and can be estimated based on the obsertation data, such as Kalman filter [14], Particle filtering [15], variable structured piece wise linear (VS-PWL) model [16], and Bayesian methods [17]. In this way, it is easier to model certain and linear behavior. The leaning-based models can learn a driver's behavior based on training from the car-following data. These models are popularly developed based on machine learning and deep learning [18, 19].

In this paper, the first order, second order and hybrid order car-following processes are defined. By comparison, some interesting findings are achieved which demonstrate significant mismatch between the existing car-following models [20,21] and real traffic data [22], when the second order car-following process is concerned. This paper provides an insight veiw on the challenges of exiting models when the higher order car-following behavior is concerned. Furthermore, the concept of hybrid order car-following behavior defined in this paper provides a guidance on the improvement of the existing car-following models, where the effects of multiple vehicles on the subject car must be considered instead of only the immediate leading one as previous models are using. A BP neural network is trained to model the hybrid order car following behavior, which validates the hybrid order idea.

\section{RELEVANT RESEARCH}

\subsection{Database for vehicle driving profles in public traffic context}

It is important to validate the mathematical and physical car-following models using real traffic data. The data set [23] is used in this paper for this purpose, which was captured on the realworld highway traffic by the next generation simulation (NGSIM) program in the Department of Transportation Federal Highway Administration, USA. The dataset contains comprehensive variables of vehicles driving on the specific highway, including the cars speed, spacing, acceleration, position, lanes, etc.

\subsection{Individual car-following models}

The earliest gazis-herman-rothery (GHR) model [24] on individual car-following behavior was reported over 60 years, based on traffic flow and driving behavior. The GHR [24] model might be the most popularly considered tool to describe the nonlinear car-following behavior between individual cars, that is, the following car and the proceeding car. Then various models have been developed inspired by this idea. The GHR model associates the acceleration or deceleration with the relative speed and space between the subject car and the leading car. As shown in (1) shows how the acceleration of the following car is determined by its own velocity and the status (the distance and the relevant speed with the leading car) in the GHR model.

$$
a_{n+1}(t+T)=\frac{C\left(V_{n+1}(t+T)\right)^{m}\left(V_{n}(t)-V_{n+1}(t)\right)}{\left(X_{n}(t)-X_{n+1}(t)\right)^{L}}
$$

where $n+1$ is the subject car, $n$ is the proceeding car, $C, m$ and $L$ are constant parameters. Since proposed, the GHR model has got various improvements, which form the family of GHR models.

The intelligent driver model (IDM) [25] describes the dynamics of the subject vehicle in (2).

$$
\begin{aligned}
& \alpha_{n+1}=\alpha\left(1-\left(\frac{v_{\alpha}}{v_{0}}\right)^{\delta}-\left(\frac{s^{*}\left(v_{\alpha}, v_{\alpha}\right)}{s_{\alpha}}\right)^{2}\right) \\
& s^{*}\left(v_{a}, v_{a}\right)=s_{0}+v_{a} T+\frac{v_{\alpha \alpha}}{\sqrt[2]{a b}}
\end{aligned}
$$

The IDM model is a superposition of acceleration and deceleration according to the desired distance $\mathrm{s}^{*}$, which is more close to the real driving behavior. Therefore, the IDM is commonly used for group vehicle interaction simulation.

\subsection{The motivation of this research}

It was commonly considered in the existing car-following models, that the driver's behavoir of the subject vehicle relies only on the status of the proceeding vehicle, which mainly include the relevant velocity and distance between the two vehicles. However, drivers do not only rely on the single vehicle immediatly proceeding the subject vehicle. There are significant impacts on the driving behavior from the status of vehicles before the proceeding one and even the ones behind the subject vehicle.

In this paper, this characteristics of driving behavior is referred as hybrid order car-following process, which means it might not be presented by a single first order model or a higher order model formed by cascading mutiple first order models. 


\section{THE PROPOSED METHOD}

This section aims to demonstrate the first order and second order model of car-following behavior. Then the agreements and disagreement are depicted between the existing models and real world cases, when the first order and the second order car-following behavior are concerned. The structural mismatches between real traffic data and existing models will be observed when the first order and the second order models are concerned. This draws forth the existence of hybrid order unmodelled dynamics in the car-following behavior, which suggests the hybrid order car-following behavior should be considered in the models.

\subsection{The first and the second order car-following behavior}

For the sake of clarity, assume three vehicles driving in the same lane as shown in Figure 1, where $\mathrm{V}$ represents the subject vehicle. V1 and V2 are the proceeding vehicles of V. From the view point of existing car-following models, V follows V1, and V1 follows V2, which forms a follower-leader chain V-V1-V2.

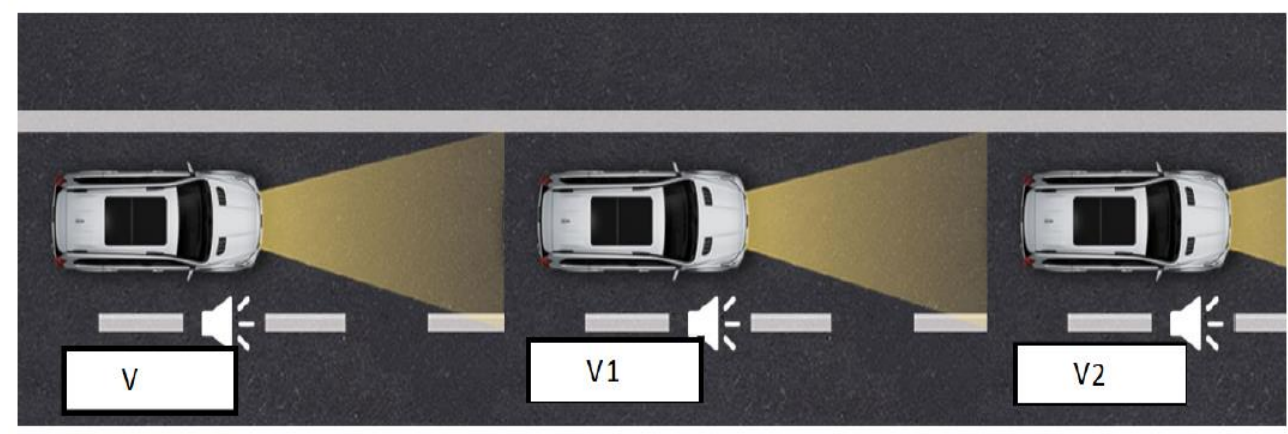

Figure 1. Proceeding vehicles and following vehicles in the same lane

A first order car-following process can be defined as the behavior of the subject vehicle following the immediate proceeding vehicle, for instance the processes of V following V1, and V1 following V2 are first order car-following behavior. From the follower-leader chain, one finds that the status(distance and relative velocity) of V2 determines the driving behavior of V1, and V1 affects V. Finally, the status of V2 are transferred to the driving behavior of V. Therefore, the second order car-following behavior describes how the subject vehicle $\mathrm{V}$ driving behavior is determined by the status of V2 (the vehicle running before its immediate proceeding vehicle). For instance, in Figure 1, the process that the status of V2 effects the driving behavior of $\mathrm{V}$ is a second order car-following process. In the similar way, even higher order car-following processes can be defined.

\subsection{Ideal second order car-following using existing models by cascading two first order processes}

This section aims to demonstrate the feasibility of using existing models to describe the higher order car-following processes, by cascading first order car-following processes. The real traffic data is used to validate the feasibility. The first experiment is to demonstrate a second order car-following behavior using the GHR model, by cascading two of such processes. The driving profile (speed and medial position in the lane) of V2 is obtained from a vehicle in real traffic. Then the GHR model is applied to determine $t$ he driving behavior of $\mathrm{V} 1$, and the $\mathrm{V} 1$ driving profile genereates the one of $\mathrm{V}$ using the same model. As shown in Figure 2(a), where the first order following process shows the distance $(\Delta \mathrm{P})$ and relative velocity $(\Delta \mathrm{V})$ between $\mathrm{V} 1$ and $\mathrm{V} 2$, and the second order following process is about the $\mathrm{V}$ and $\mathrm{V} 2$. From Figure 2(a), one finds the status transformation from V1 to V and the one from V2 to V are closely relevant(the similar shape of $\Delta \mathrm{P}-\Delta \mathrm{V}$ curves). In fact, in the second order following process, the subject vehicle $\mathrm{V}$ just enlarges the range of status deviation, while keeping the basic driving profiles of V2.

The second experiment considers the commonly recognized IDM model. Figure 2(b) shows the first order and the second order following processes (V1-V2 and V-V2 respectively) in IDM model. Similar to the GHR model, the transformation of vehicle status in the second order following process (V-V2) enlarges the first order following behavior (V1-V2) but keeping the characteristics of the driving profile. 


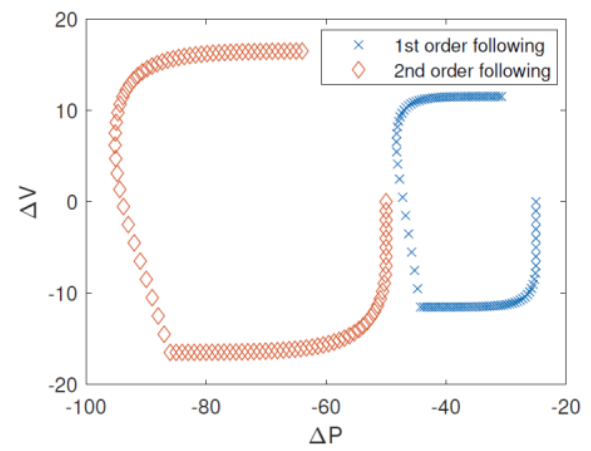

(a)

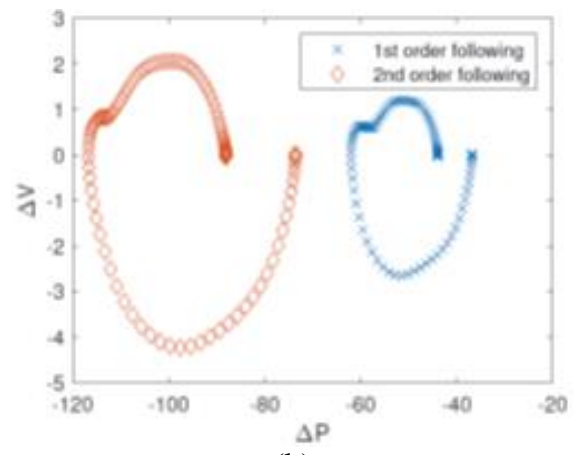

(b)

Figure 2. Ideal first order and cascade second order following processeses, (a) GHR model, (b) IDM model

\subsection{Some real world cases support the ideal cascading first order following processes}

After demonstrate the vehicle status transformation in second order car-following behavior based on models, this section aims to validate if the similar phenomena is supported by real world data as well. In this way, we can validate the feasibility of the models. Towards to this purpose, the data set [22] is utilized. The identity number of a vehicle in the data set is used to identify the vehicle, for example, \#1 means the vehicle in the data set having the ID number 1. The follower-leader relationship between the subject vehicle and the proceeding vehicle is extracted from the data set. Then the relevant velocity $(\Delta \mathrm{V})$ and distance $(\Delta \mathrm{P})$ are calculated between a vehicle and its immediate proceeding vehicle (for the first order following process), and the vehicle immediately proceeding the proceeding one (for the second order following process).

Firstly, some cases are demonstrated that strongly support the ideal second order following by cascading first order following processes. In the data set, the vehicle with ID 36(denoted as vehicle \#36, the same for the rest of the paper) are following vehicle \#24, and \#24 following \#21, they form the followerleader chain \#36-\#24-\#21. For details about the data please refer to the data set. Very similar relationship is discovered as we did using the ideal models, where vehicle \#36 is closely determined by \#24 and \#24 closely relies on \#21, so all of them are keeping simple driving profile as shown in Figure 3(a). Figure 3(b) shows more complex following behavior in the follower-leader chain \#95-\#170-\#166. The vehicle relevant status also keeps high similarity. In Figure 5, the second order following shows the typical enlarging characteristics as the findings obtained in the ideal models.

To make the status transfermation more clear, the follower-leader chain \#63-\#50-\#39 is selected where the first order and the second order following processes are relatively far. As shown in Figure 4(a), one finds the distribution of the $\Delta \mathrm{P}-\Delta \mathrm{V}$ observations are fairly enlarged with similar scale. All of these experiments show the strong link between the first order following and the second order following processes. In these cases, the existing models do well match with the real traffic data.

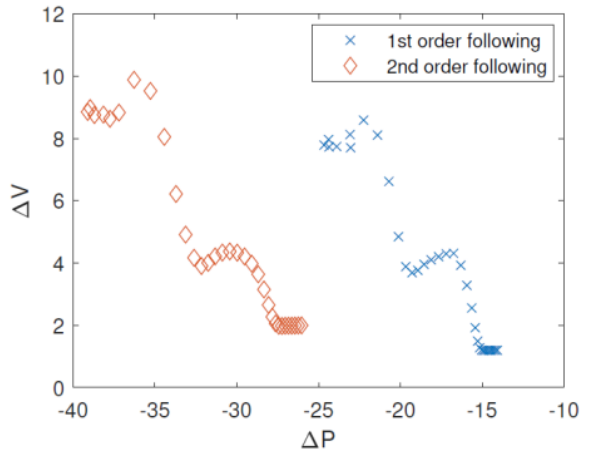

(a)

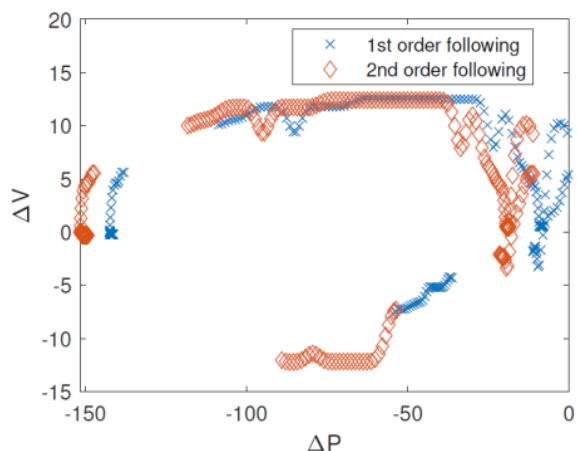

(b)

Figure 3. Frist order and second order following behaviours, (a) a simple example, (b) a complex example 


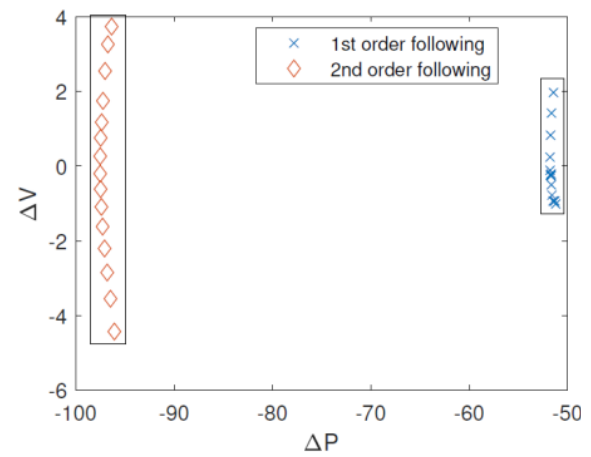

(a)

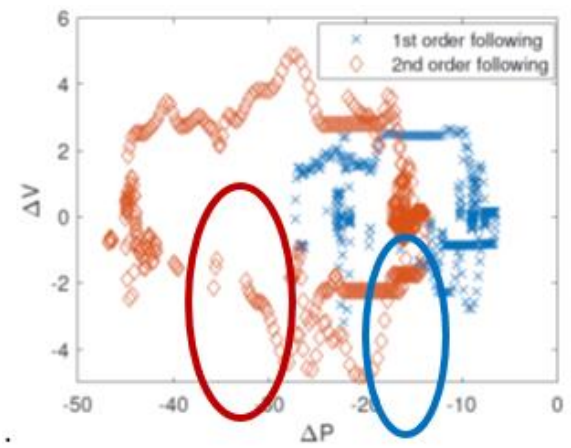

(b)

Figure 4. The status transfermation between the1st order and the 2 nd order following processes, (a) matched transfermation, (b) dismatched transfermation

\subsection{Structural mismatching of models in real world data}

While some real world cases support the cascading first order car-following processes, there are more cases against this concept by significant mismatches between the first order and the second order following processes. This section aims to depict some of these cases, and provide some clues of possible reasons of the mismatches. Figure $4(\mathrm{~b})$ depicts the relevant driving profiles $(\Delta \mathrm{P}-\Delta \mathrm{V})$ of the follower-leader chain \#59-\#39-\#17, where the left half of the first order and the second order following profiles (the sections encirculated) show significant structural mismatch, although the right parts have some similarity. This observation means there must exist other factors significantly affect the driving behavior of the subject vehicle (\#59), for instance, the impacts from other vehicles instead of the immediate proceeding one only. Figure 5(a) and Figure 5(b) show even more and stronger structural mismatches.

Such observations are commonly supported by the concerned data set. It is very interesting, because it implies that by cascading multiple first order following processes cannot describe real world second or higher order car-following behavior in most cases. Therefore, the higher order car-following model should be reconsidered to address these structural mismatch.

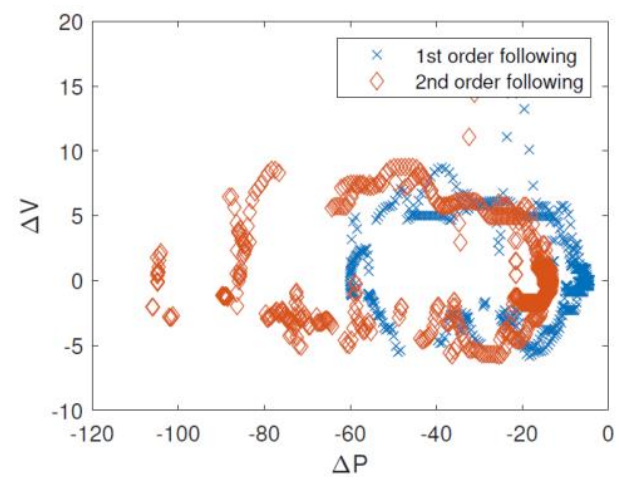

(a)

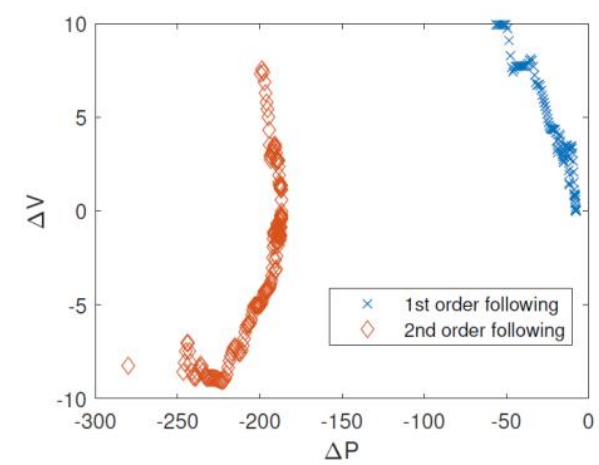

(b)

Figure 5. Car following behavior with unmodelled dynamics, (a) a complex case, (b) a simple case

\subsection{Hybrid order uncertainties}

Except for the cases supporting the cascading first order car-following processes, more popular cases containing significant structural mismatches between the first order and the second order following modes. These mismatches hint the existence of other factors that affect the driving behavior of the subject vehicle, which cannot be modelled by cascading multiple first order following processes. Furthermore, the effect from each factor are not supposed to be linearly independent. Therefore, hybrid order dynamics exist in the car-following behavior, which need deeper insight analytic model. A production from nonlinearly dependent impacts from more vehicles and other factors, such as the road condition and comprehensive ambient influences, are needed for reasonably modelling the complex car-following nature. 
A simplificaiton can be considered for the purpose of reasonably modelling the existence of multiple factors affect the driving profile, by containing only vehicles driving close to the subject vehicle, without other ambient influences. For instance, the contributions from multiple order following behavior (the subject vehicle determines the driving profile by considering multiple vehicles around) should be considered in such model, whereby the hybrid order concept embeds. In such model, the subject vehicle driving profile should be a mixture of different order models.

\subsection{A neural network model for hybrid order car following behavior}

From the structual mismatch of existing car following models, the hybrid order behavior is observed. Multiple parameters should be considered to detemine the driving behavior of the subject vehicle, instead of only the driving profile of the immediate proceeding vehicle. This bybrid order behavior can be represented by a multiple variable function shown in the (4).

$$
a=f\left(p, p_{1}, p_{2}, v, v_{1}, v_{2}, \ldots\right)
$$

where $a, p$ and $v$ are is the acceleration, position and velocity of the subject vehicle, respectively.

The subscript 1 and 2 indicate the immediate proceeding vehicle and the one even proceeding, respectively. In fact, there can be many more varialbes considered in this function, that is, any factors actualy affect the driving behavior of the subject vehicle should be included. The $p, p_{1}, p_{2}$ can also be replaced by the distances between vehicles.

As the list of parameters in the function of (4), it is difficult to explicitly derive the driving behavior analisticly. This paper employs a 3-layer backpropagration nuerual network to model the proposed hybrid order behavior, with the purpose of simply demonstrate the potential improvement if the idea of hybrid order can be introduced to the car following behavior modelling. Figure 6 depicts the 3-layer neural network with 6 input, 10 hidden neurons, the hidden neurons have sigmoid functions. The algorithm indicated. The Levenberg-Marquardt training algorithm is used to train the network. Obviously, this neural network only aims to validate the hybrid order idea. More variables should be considered for more reliable performance. This paper only uses the driving profiles of the immediate proceeding vehicle and its proceeding one. In fact, other vehicles, road condition, even the driver's driving habit and mental status should be considered.

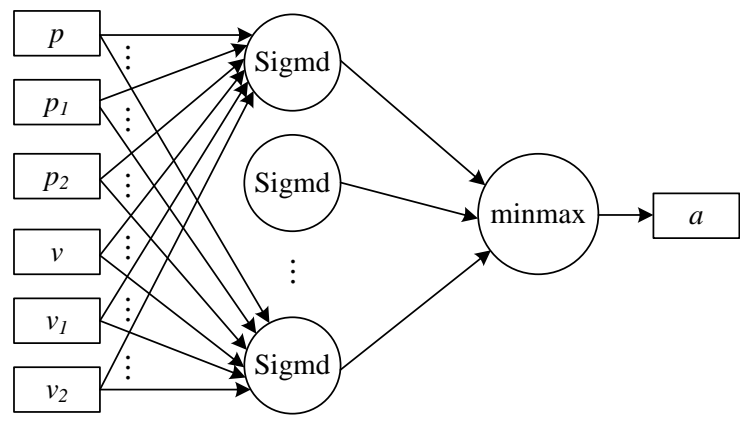

Figure 6. The BP network modelling hybrid order behavior

\section{RESULTS AND DISCUSSION}

The real traffic data from the data set [22] is used to validate the modelling capcity of the second order GHR model and the proposed hybrid order model. The accuracy of prediction on the subject vehicle velocity and position is used as the performance evaluating the different models. The acceleration of the subject vehicle is predicted by these models and then integrated to obtain the velocity and position.

Experiments are designed by considering the different driving styles commonly experienced, including speeding up, maining speed, braking and urgent braking. The real velocity of the leading vehicle is used as the ground truth, which is used to validate the prediction capacity of the compared models. Figures 7(a) and 7(b) demonstrate the prediction of driving velocity and position of the subject vehicle. From Figure 7, one finds that the hybrid order model captured the pattern of velocity and position, with smaller error to the ground truth.

The experiment for a speed maintaining process is depicted in Figures 8(a) and 8(b). In a speed maintaining process, the driver usually tries to keep the velocity varying in a relevantly small range. As shown in Figure 8, the velocity of the subject vehicle varies in the range between 25 to 35 feet per second. The velocity values show viberating pattern. The hybrid model captured this pattern, however second order 
model of the GHR method just follows a smoothed envelope of the velocity viberation. Furthermore, the hybrid order model accurately predicted the subject vehicle position, although the GHR model also predict the position with a good performance. From the comparison in Figures 8(a) and 8(b), it is reasonable to consider that the driving profile will be affected by more variables, because the driver has higher possibility to concern with other vehicles. Therefore, the driving profile can be affected.

The third experiemnt is for the process of braking. As shown in Figure 9(a), the subject vehicle reduced the velocity from 27 to 22 feet per second in 0.9 seconds. The hybrid order model got the trend and values of the velocity, but the GHR model did not. Again, it means the two proceeding vehicles affect the subject vehicle driving profile in a hybrid order style. The position shown in Figure 9(b) was predicted accurately as well.

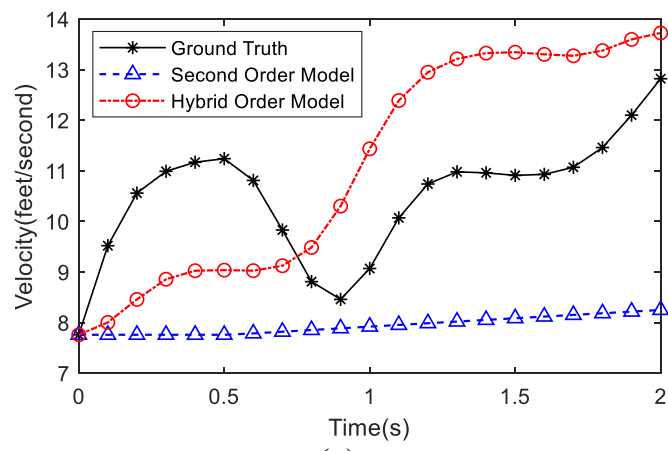

(a)

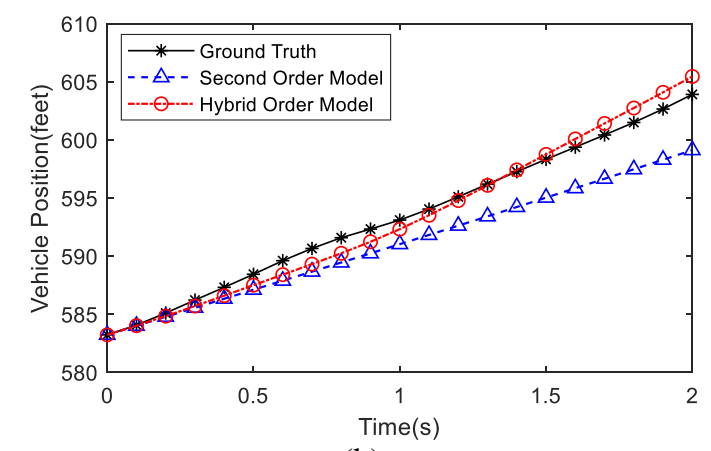

(b)

Figure 7. The car following status prediction during speeding up process, (a) velocity, (b) position

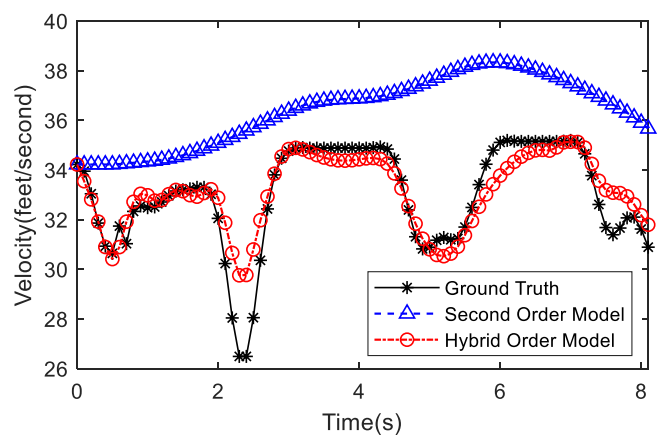

(a)

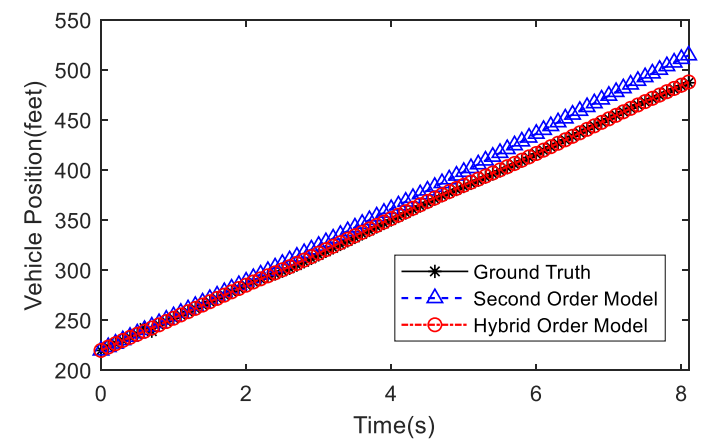

(b)

Figure 8. The comparison of subject vehicle status prediction during speed maintaining process, (a) velocity, (b) position

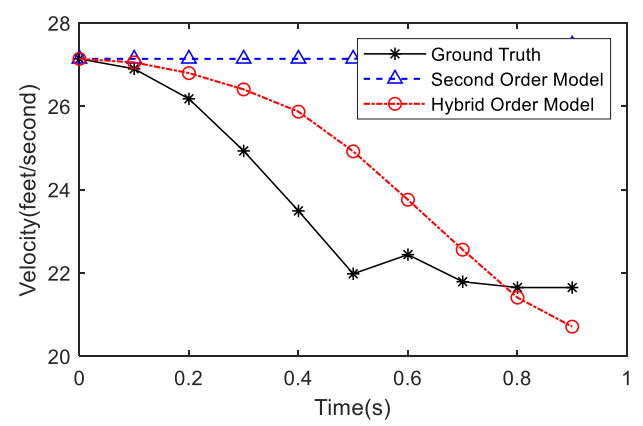

(a)

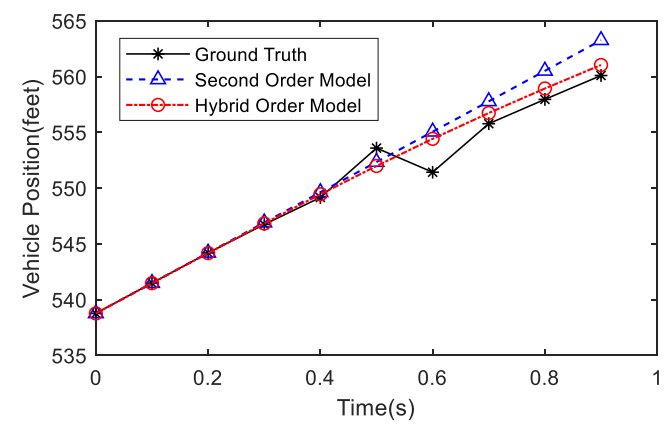

(b)

Figure 9. The comparison of subject vehicle status prediction during braking process, (a) velocity, (b) position 
When urgent braking is concerned, the last experiment was designed. Because a urgent braking process is closely related to the driving habit and decision making style of the driver. The driving profile under this situation, might have more effects from the mental behavior, so from the proceeding vehicles it is more difficult to accurately predict when compared to other driving scenarios. For instance, as shown in Figure 10(a), the driving speed dropped more than 23 feet per second within 1 second (from 0.5 th second to 1.5th second). Figures 10(a) and 10(b), the prediction of the subject vehicle velocity and position, indicate the significant error in both models, but the proposed hybrid order model performs better.

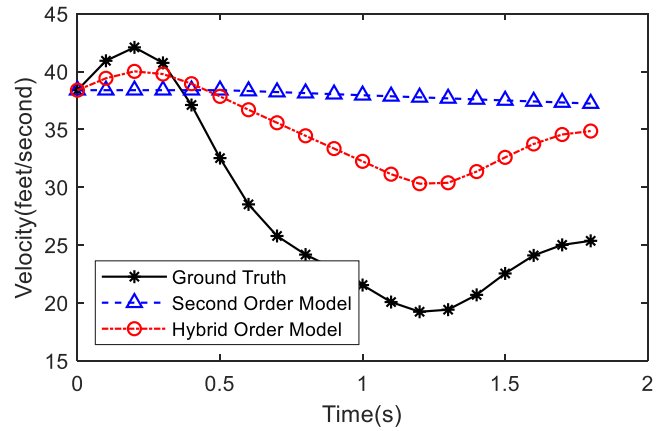

(a)

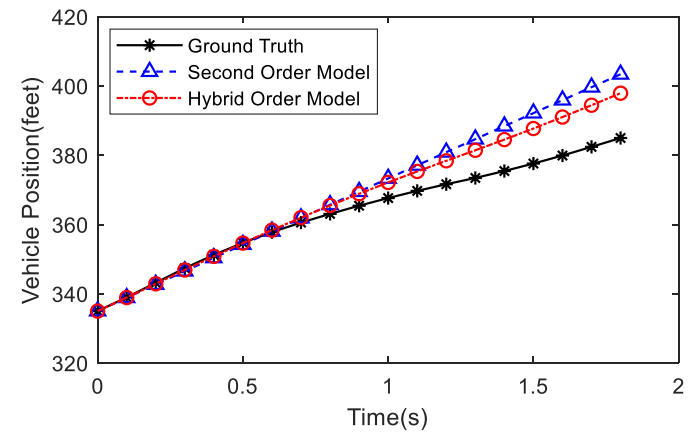

(b)

Figure 10. The vehicle velocity prediction during urgent braking process, (a) velocity, (b) position

\subsection{Discussion on the experiments and results}

From the real traffic data, 4 commonly experienced scenarios were selected and demonstrated in experiments. Significant improvments were observed after the hybrid order idea is applied. These improvements suggest the existence of hybrid order behavior. In another way, the variables affecting the driving profiles can be different with the scenarios, which means it is necessary to use data driven method to capture the actual behavior of the driver(for example the neural networks or other machine learning mehtods).

\section{CONCLUSION}

This paper proposed the concepts of the order of car-following behavior. The first order, the second order, and the hybrid order following processes were defined. These concepts were applied to both the ideal models and real world traffic data. The hybrid order following behaviors were observed in the real traffic, where the existing models generated significant mismatches with the real traffic data. The hybrid order model provides a space for modelling the car-following behavior in the view point of wider scope to choose vehicles having impacts on the subject vehicle, which has both theoretical and practical values for macro and micro vehicle behavior analysis. A BP network was employed to simply demonstrate the improvement on the driving profile prediction, when the hybrid order idea is applied. The future work would be the analytic mathematical modelling of such hybrid order car-following model and the utilization in the intelligent traffic system

\section{REFERENCES}

[1] Bando, M., et al., "Phenomenological study of dynamical model of traffic flow," Journal de Physique I, vol. 5, no. 11, pp.1389-1399, 1995.

[2] Jimnez, F., et al., "Advanced driver assistance system for road environments to improve safety and efficiency," Transportation research procedia, vol. 14, pp. 2245-2254, 2016.

[3] Bernhardt, K. L., Roeder, W. H. and Chamberlin, J., "Hand Held Products Inc.: Collision avoidance system and method," U.S. Patent Application, vol. 14, no. 880, 2016.

[4] Shehan, M. A., Snarski, K., Mackey, J., Mc Connell, D. and Kanzler, S. G., "Ford Global Technologies LLC: Vehicle blind spot system operation with trailer tow," U.S. Patent, vol. 9, no. 493, 2016.

[5] Pipes, L. A., "An operational analysis of traffic dynamics," Journal of applied physics, vol. 24, no. 3, pp. 274-281, 1953.

[6] Newell, G. F., "Nonlinear effects in the dynamics of car following," Operations research, vol. 9, no. 2, pp. 209-229, 1961.

[7] Rovira E., et al., "Looking for Age Differences in Self-Driving Vehicles: Examining the Effects of Automation Reliability, Driving Risk, and Physical Impairment on Trust," Front in Psychology, vol. 10, no. 800, 2019.

[8] Schwarting, J., et al., "Social behavior for autonomous vehicles," PNAS (Proceedings of the National Academy of Sciences of the United States of America), vol. 116, no. 50, pp. 24972-24978, 2019. 
[9] Gupta, S., et al., "Pedestrian's risk-based negotiation model for self-driving vehicles to get the right of way," Accident Analysis \& Prevention, vol. 124, pp. 163-173, 2019.

[10] Gipps, P. G., "A behavioural car-following model for computer simulation," Transportation Research Part B: Methodological, vol. 15, no. 2, pp. 105-111, 1981.

[11] Zhao, D., et al., "Accelerated Evaluation of Automated Vehicles in Car-Following Maneuvers," arxivId 1607.02687, 2016.

[12] Kesting, A., Treiber, M. and Helbing, D., "General lane-changing model MOBIL for car-following models," Transportation Research Record: Journal of the Transportation Research Board, vol. 1999, no. 1, pp.86-94, 2007.

[13] Wang, W., et al., "Development and evaluation of two learning-based personalized driver models for car-following behaviors," 2017 American Control Conference (ACC), Seattle, WA, pp. 1133-1138, 2017.

[14] Li, Y., et al., "Fusion Modeling Method of Car-Following Characteristics," IEEE access, vol.7, pp. 162778-162785, 2019.

[15] Kasai, M., Xing, J., "Use of Particle Filtering to Establish a Time-Varying Car-Following Model," International Journal of Intelligent Transportation Systems Research, vol. 17, pp. 49-60, 2018.

[16] Nwadiuto J., et al., "Analysis and Modeling of Real World Car-Following Driving in Downtown Area Based on Variable Structured Piecewise Linear Model," 2019 IEEE Intelligent Transportation Systems Conference (ITSC), Auckland, New Zealand, pp. 1211-1216, 2019.

[17] Zhao, M., et al., "A Car-Following Model Considering Preceding Vehicle's Lane-Changing Process," IEEE Access, vol. 7, pp. 89913-89923, 2019.

[18] Chen, C., et al., "Deepdriving: Learning affordance for direct perception in autonomous driving," 2015 IEEE International Conference on Computer Vision (ICCV), Santiago, pp. 2722-2730, 2015.

[19] Wang, X., et al., "Capturing car-following behaviors by deep learning," IEEE Transactions on Intelligent Transportation Systems, vol. 19, no. 3, pp. 910-920, 2018.

[20] Gazis, D. C., Herman, R. and Rothery, R. W., "Nonlinear Follow-The-Leader Models of Traffic Flow," Operations Research, vol. 9, no. 4, pp. 545-567, 1961.

[21] Treiber, M., Hennecke, A., Helbing, D., "Congested traffic states in empirical observations and microscopic simulations," Physical Review E, vol. 62, pp. 1805-1824, 2000.

[22] “US Highway 101 Dataset,” 2019. [Online], Available: https://www.fhwa.dot.gov/publications/research/operations/07030/.

[23] Nakayama, A., et al., "Quantitative explanation of circuit experiments and real traffic using the optimal velocity model," New Journal of Physics, vol. 18, 2016.

[24] Jiang, R., Wu, Q. and Zhu, Z., "Full velocity difference model for a car-following theory," Physical Review E, Statistical, nonlinear, and soft matter physics, vol. 64, no. 1, p. 017101, 2001.

[25] Al-Jameel, "Examining and improving the limitations of Gazis-Herman-Rothery car-following model," Conference Salford Postgraduate Annual Research Conference, The University of Salford, Salford, United Kingdom, 2009.

\section{BIOGRAPHIES OF AUTHORS}

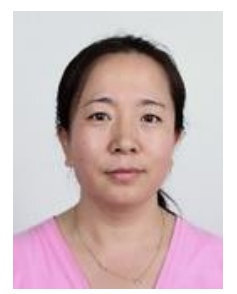

Dr Chunling Tu received the Bachelor degree of computer science from Tianjin University of Technology and Education, China in 2002; MTech and MSc degrees in Electrical Engineering from Tshwane University of Technology (South Africa) and ESIEE Paris University (France) in 2010; DTech and $\mathrm{PhD}$ degrees of Electrical Engineering from Tshwane university of Technology and University Paris East, France in 2015. She is currently a senior lecturer at Tshwane University of Technology. Her research interests include image processing, AI, industrial control, machine learning, deep learning and pattern recognition.

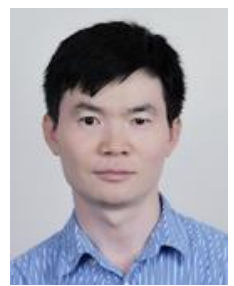

Shengzhi Du received the M.S. degree in control theory and control engineering from Tianjin Poly Technology University, Tianjin, China, in 2001 and the Ph.D. degree in control theory and control engineering from Nankai University, Tianjin, China, in 2005. He is currently a professor in French South Africa Institute of Technology (F'SATI), Tshwane University of Technology, South Africa. His research interests include computer vision, AI, pattern recognition, and Human in the loop systems. 